a mammona et a baccho initium habet. Habebimus, timeo, acriora dissidia, mortuo hoc comite. Confluunt undique evangelistae, suadentes super communione utriusque speciei sed, ut audio, hactenus renuit.

Ediert (in lateinischer übersetzung) von Johannes Cochläus in dem schriftchen: Ex compendio Actorum Martini Lutheri caput ultimum, Moguntiae I548, unter dem titel: Ex epistola germanica cuiusdam civis mansfeldensis narratio historica de ultimis Martini Lutheri actis et eius obitu; desgleichen in seiner Historia de actis et scriptis Martini Lutheri etc. Paris I565 S. $299 \mathrm{ff}$. Der Nachweis, dass der "cives Mansfeldensis" der to Eislebener apotheker Johann Landau war, bei N. Paulus, Luthers Lebensende und der Eislebener Apotheker Johann Landau, Mainz 1896.

\title{
VERZEICHNIS DER ABGEDRUCKTEN AUTHENTISCHEN BERICHTE UBBER LUTHERS LETZTE LEBENSSTUNDEN
}

I. Schreiben des Dr. Justus Jonas (Superintendent in Halle) an Kurfürst Johann Friedrich von Sachsen (in Torgau). Eisleben I546, Febr. 18

2. Brief des Grafen Albrecht von Mansfeld an den Kurfürsten Johann Friedrich von Sachsen. Eisleben I546. Febr. I8. . . .

3. Brief des Fürsten Wolfgang von Anhalt an den Kurfürsten Johann Friedrich von Sachsen. Eisleben 1546, Febr. 18 . . .

4. Brief des Magister Johannes Aurifaber an Michael Gutt (Kammerschreiber in Halle). Eisleben 1546, Febr. 18.

5. Brief des Grafen Hans Georg von Mansfeld an Herzog Georg von Sachsen (in Freiberg). Eisleben 1546, Febr. I 8

6. Brief des Eislebener Ratsherrn Andreas Friedrich an [seinen Onkel] Johann Agricola [Hofprediger in Berlin]. Eisleben 1546, Febr. 18

7. Melanchthon teilt seinen Zuhörern den Tod Luthers mit. Witten-

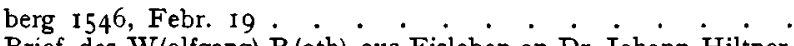

8. Brief des W(olfgang) $\mathrm{R}$ (oth) aus Eisleben an Dr. Johann Hiltner, Syndicus zn Regensburg. Eisleben I546, Febr. 19. . . .

9. Die Todesbeschreibung in der Trauerpredigt des Michael Coelius, gehalten am 20. Februar in der S. Andreaskirche zu Eisleben, wo Luthers Leiche aufgebahrt war · Abshrift der Beilage zu einem Brief des Grafen Hans Georg

Io. Abschrift der Beilage zu einem Brief des Grafen Hans Georg
von Mansfeld an Georg von Selmenitz (in Wittenberg). Eisleben 1546, Febr. 22.

I I. Bericht, den Prof. Spaeth-Philadelphia auf dem letzten Blatt und der Innenseite des Deckels einer zu Wittenberg im Jahre I544 gedruckten Postille seiner Seminarbibliothek fand . . . .

12. Beilage zu einem lateinischen Briefe des Andreas Münzer an Herzog Albrecht von Preussen. (Der brief ist datiert Witten-

13. Brief des Dr. Justus Jonas an Veit Dietrich (Prediger in Nürnberg). Halle 1546 , März 9. . . . . . . . . . . 23 
I4. "Vom christlichen abschied aus diesem tödlichen leben, des ehrwirdigen herrn D. Martini Lutheri, bericht, durch D. Justum Jonam, M. Michaelem Celium, unnd ander die dabey gewesen, kurtz zusamengezogen" . . . . . . . . . . . . 24

I5. Brief des Dr. Justus Jonas an König Christian III von Dänemark (in Kolding). Halle I546, April I5 . . . . . . . . 31

I6. Bericht des Eislebener Apothekers Johann Landau an (seinen Vetter) Georg Wizel in Regensburg, verfasst vor dem 6. Juni 1546

\section{PERSONENREGISTER}

Dr. Martin Luther, Dr. Justus Jonas und Magister Michael Coelius, deren namen fast auf jeder seite vorkommen, sind nicht mit in das register aufgenommen

Agricola, Johann, hofprediger in Berlin 9

Albrecht, Hans, stadtschreiber in Mansfeld 4. 5. 15. 17. 19. 28 seine frau 4. 5. 15. 17. 19. 28

Amsdorf, Nikolaus von, evangelischer bischof zu Naumburg.Zeitz 6

Anhalt, Fürst Wolfgang von 3. 7. 8. I5. I9. 21. 22. 30. 37

Aurifaber, Johann, magister 8. 15. I7. 19. 20. 22. 26. 27. 28

Baumgartner 24.

Brück, Dr. Georg, sächsischer kanzler 12

Bugenhagen, Dr. Johann, gen. Pomeranus, prediger zu Wittenberg 5. I 2

Cochlaeus, Johann 40

Cruziger (Creuziger, Creuzinger etc. geschrieben), Kaspar 5. I 2. 35

Dietrich, Veit, prediger zu Nürnberg 23. 24. $30 \mathrm{anm}$.

Dänemark, König Christian III, von 31.32

Drachstedt, Dr. 30. 38

Ebner, E. 24

Friedrich, Andreas, ratsherr zu Eisleben $9 \mathrm{f}$.

Gasman, Hans, Honsteinischer rentmeister zu Ellrich 29

Gutt, Michael, kammerschreiber in Halle 8

Hiltner, Dr. Johann, syndicus in Regensburg $12 \mathrm{ff}$.

Jonas, frau Dr. Justus 32

deren kinder 32

Justus jr. 32

Khol, Hans, buchdrucker in Regensburg 6

Landau, Johann, apotheker in Eisleben $33.36 \mathrm{ff} .40$

Lange, Dr. Johann, prediger in Erfurt 2 anm.

Ludwig, Dr., arzt in Eisleben 4. 5. 8. 17. 20. 22. 28. 29. 36

Luther, frau Dr. Martin 3. 29. 32. 39

deren söhne Martin und Paul 3. 4. I4. 26. 27

Magenbuch 24

deren kinder Johannes und Margarete 32

Major, Dr. Giorg 6

Mansfeld, die Grafen von 4. 8. 13. 15. 24. 26. 27. 31. 33. 37. 38

Graf Albrecht 3 ff. 7. 8. I1. I4. 15. 17. I9 ff. 27 ff. $3^{6}$ dessen gemahlin 4. 8. I I. I 4. I5. I7, I9 ff. $27,28 \mathrm{ff}$.

Philipp 8. 21. 22. 30

"Hans Georg 8. 9. 18. 21. 22. 30

, Volrath 8. 2 I. 22. 30

" Gebhardt 2 I

dessen gemahlin $\mathbf{2} \mathbf{I}$ 